\title{
IMPLEMENTATION OF ENO AND WENO SCHEMES FOR FINITE VOLUME UNSTRUCTURED GRID SOLUTIONS OF COMPRESSIBLE AERODYNAMIC FLOWS
}

\begin{abstract}
W. R. Wolfa and J. L. F. Azevedob

${ }^{a}$ Instituto Tecnológico de Aeronáutica

Departamento de Engenharia Eletrônica e de Computação

CP. 12228-900, São José dos Campos, São Paulo, Brasil

wolf@ita.br

$\mathrm{b}^{\mathrm{b}}$ Instituto de Aeronáutica e Espaço

Divisão de Sistemas Espaciais

CP. 12228-904, São José dos Campos, São Paulo, Brasil

ABSTRACT

In this work the essentially non-oscillatory schemes (ENO) and the weighted essentially non-oscillatory schemes (WENO) are implemented in a cell centered finite volume context on unstructured meshes. The 2-D Euler equations will be considered to represent the flows of interest. The ENO and WENO schemes have been developed with the purpose of accurately capturing discontinuities appearing in problems governed by hyperbolic conservation laws. In the high Mach number aerodynamic studies of interest in the present paper, these discontinuities are mainly represented by shock waves and contact discontinuities. The entire reconstruction process of ENO and WENO schemes is described in detail for linear polynomials and, therefore, second-order of accuracy. An extension to higher orders of accuracy is presented in the paper in a straightforward manner and applications for compressible flows are shown. These applications compare the accuracy of the schemes with some related data that appear in the references cited in this paper or that come from analytical solutions.
\end{abstract}

Keywords: ENO, WENO, CFD, Unstructured Grids, Finite Volume

\section{NOMENCLATURE}

A Roe's flux Jacobian matrix

$c \quad$ non dimensional speed of sound

$e \quad$ non dimensional total energy per unit of volume

$E, F$ flux vectors in the $(\mathrm{x}, \mathrm{y})$ cartesian directions, respectively

$G \quad$ Gaussian point

$p \quad$ non dimensional pressure

$Q \quad$ vector of conservative properties

$S \quad$ surface of the control volume

$t \quad$ non dimensional time

$u, v \quad$ non dimensional velocities in the $(\mathrm{x}, \mathrm{y})$ cartesian directions, respectively

$V \quad$ control volume

w Gaussian weight

\section{Greek symbols}

$\alpha \quad$ coefficients used in the Runge-Kutta scheme

$\gamma \quad$ ratio of specific heats

$\Gamma \quad$ edge of the control volume

$\rho \quad$ non dimensional density

\section{Subscripts}

i i-th volume

ng ng-th neighbor of the i-th volume

\author{
Superscripts \\ n n-th iteration
}

\section{INTRODUCTION}

In this work, the essentially non-oscillatory schemes (ENO) introduced by Harten et al. (1987), and the weighted essentially non-oscillatory schemes (WENO) introduced by Liu et al. (1994), are implemented in a cell centered finite volume context for unstructured meshes. The two dimensional Euler equations will be considered to represent the flows of interest. The ENO and WENO schemes have been developed with the purpose of accurately capturing discontinuities appearing in problems governed by hyperbolic conservation laws. In the high Mach number aerodynamic studies of interest in the present paper, these discontinuities are mainly represented by shock waves and contact discontinuities. The entire reconstruction process of ENO and WENO schemes is described in detail for linear polynomials and, hence, second-order of accuracy, with extension to higher orders, and applications to compressible flows are presented showing the accuracy of the schemes and their behaviour. For the ENO schemes, interpolation polynomials of one order less than the order of accuracy expected in the solution are computed and these polynomials are a good approximation to the values of the conserved variables within the cells. These polynomials interpolate primitive variable values in Gauss quadrature points using stencils determined by a Von Neumann neighborhood (Sonar, 1997). The control volume moments and the mean 
values of primitive variables in the cells are used to compute the polynomial coefficients and, hence, one can compute the oscillation of the polynomials and select the smoothest among them through the values of these coefficients. While the ENO schemes use the smoothest polynomial, the WENO schemes use all ENO computed polynomials for the stencils and, therefore, they construct one polynomial only. Non negative weights, which must add up to one, are computed for every polynomial through oscillation indicators and the WENO polynomial is constructed by the sum of all the ENO polynomials multiplied by the respective weights (Liu et al., 1994, Friedrich, 1998). The weights attributed to the polynomials can be of the order of accuracy desired in the solution if these polynomials are in the discontinuous regions of the mesh or they can be of order one if the polynomials are in the smooth regions of the flow. Classical computational fluid dynamics applications such as shock tube problems, the flow in a channel with a forward-facing step and the transonic flow over a NACA0012 are presented with the aim of comparing the accuracy of the proposed methods. Such an assessment of the methods here implemented is achieved through the comparison of the present numerical results with analytical solutions, with the results obtained with different second-order methods already tested and validated by the present research group, and with other independent computational data available in the literature.

\section{THEORETICAL FORMULATION}

\section{Governing equations}

In the present work, the 2-D Euler equations are solved in their integral form as Eq. (1),

$$
\frac{\partial}{\partial t} \int_{V} Q d V+\int_{V}(\nabla \cdot \vec{P}) d V=0
$$

where $\vec{P}=E \hat{i}+F \hat{j}$. The application of the divergence theorem to Eq. (1) yields to

$$
\frac{\partial}{\partial t} \int_{V} Q d V+\int_{S}(\vec{P} \cdot \vec{n}) d S=0
$$

where $V$ represents the control volume, $S$ represents the surface of the control volume and $\vec{n}$ is the unit normal vector outward to the surface, $S$, positive outward. The vector of conserved variables, $Q$, and the convective flux vectors, $E$ and $F$, are given by

$$
Q=\left\{\begin{array}{l}
\rho \\
\rho u \\
\rho v \\
e
\end{array}\right\}
$$

$$
E=\left\{\begin{array}{l}
\rho u \\
\rho u^{2}+p \\
\rho u v \\
(e+p) u
\end{array}\right\} F=\left\{\begin{array}{l}
\rho v \\
\rho u v \\
\rho v^{2}+p \\
(e+p) v
\end{array}\right\} .
$$

In the equations above, $\rho$ represents the density, $p$ is the pressure, $u$ and $v$ represent the Cartesian components of the velocity, and $e$ is the total energy per unit of volume. The system is closed by the equation of state for a perfect gas

$$
p=(\gamma-1)\left[e-\frac{1}{2} \rho\left(u^{2}+v^{2}\right)\right]
$$

where the specif heat ratio $\gamma$ was choosen as 1.4 for all the computations in this work.

Equation (2) discretized in a cell centered finite volume context can be rewritten for the $i-t h$ control volume as Eq. (6)

$$
\frac{\partial Q_{i}}{\partial t}=-\frac{1}{V_{i}} \int_{S}(\vec{P} \cdot \vec{n}) d S,
$$

where $Q_{i}$ is the mean value of $Q$ at time $t$ over the $i-t h$ control volume $V_{i}$.

\section{NUMERICAL FLUX EVALUATION}

The spatial discretization is concerned in finding a discrete approximation to the integral in the right side of Eq. (6). The control volumes considered in this work are the triangles themselves and they can be decomposed into a finite number of line segments $\Gamma_{j}$. One should observe that the control volumes could be composed by any type of polygon, such as quadrilaterals for example. In this work, as an evolutionary step, the authors have restricted themselves, however, to triangular grids. Hence, it is possible to write

$$
S_{i}=\bigcup \Gamma_{j}
$$

Thus, the boundary integral from Eq. (6) can be decomposed into

$$
\int_{S}(\vec{P} \cdot \vec{n}) d S=\sum_{j} \int_{\Gamma_{j}}(\vec{P} \cdot \vec{n}) d S,
$$

and, as $\vec{n}$ is constant on each line segment $\Gamma_{j}$, the right side of Eq. (8) is discretized using $N$-point Gaussian integration formulae with degree $2 N-1$ and order of accuracy $2 N$. Using the integration formulae cited above, one obtain the following approximation

$$
\int_{S}(\vec{P} \cdot \vec{n}) d S \approx \sum_{j}\left|\Gamma_{j}\right| \sum_{l=1}^{N} w_{l} \vec{P}\left(Q\left(G_{l}\right), t\right) \cdot \vec{n},
$$


where $G_{l}$ and $w_{l}$ are, respectively, the Gaussian points and the weights on line segment $\Gamma_{j}$.

Using the method described above one can compute

values to $Q_{i}$ in some instant $t$ and then, from these mean values, one can reconstruct polynomials that represent the primitive variables $\rho, u, v$ and $p$. Finally, it is possible to compute values of the conserved variables in the Gaussian points. Due to the discontinuity of the reconstructed values of the conserved variables over the cell boundaries, one must use a numerical flux function to approximate these values on the cell boundaries.

In this work, the authors have used the Roe flux difference splitting method (Roe, 1981) to compute this approximation. Hence, the flux evaluation in the right hand side of Eq. (9) is computed as

$$
\begin{aligned}
& \vec{P}\left(Q\left(G_{l}\right), t\right) \cdot \vec{n} \approx \vec{P}\left(Q_{i}\left(G_{l}\right) ; Q_{n g}\left(G_{l}\right), t ; \vec{n}\right)= \\
& \frac{1}{2}\left[\vec{P}\left(Q_{i}\left(G_{l}\right)\right)+\vec{P}\left(Q_{n g}\left(G_{l}\right)\right)\right] \cdot \vec{n} \\
& -\frac{1}{2}\left|A\left(Q_{i}\left(G_{l}\right) ; Q_{n g}\left(G_{l}\right), t ; \vec{n}\right)\right|\left[\left(Q_{i}\left(G_{l}\right)\right)-\left(Q_{n g}\left(G_{l}\right)\right)\right]
\end{aligned}
$$

In Eq. (10), the $A$ matrix is a positive semidefinite matrix formed from the flux Jacobian matrix in the sense defined by Roe, (1981). In the same equation, $Q_{i}\left(G_{l}\right)$ is the vector of conserved variables computed at the quadrature point, $G_{l}$, using the polynomial reconstruction associated to the $i-t h$ control volume. Similarly, $Q_{n g}\left(G_{l}\right)$ is the vector of conserved variables computed at the same quadrature point, $G_{l}$, but using the polynomial reconstruction associated with the $n g-t h$ control volume. Here, it is being assumed that " $n g$ " represents the neighbor of the $i$-th control volume, which shares the $\Gamma_{j}$ line segment with the $i-t h$ cell.

\section{TEMPORAL DISCRETIZATION}

In the present work, the system of equations to solve became an ordinary differential equation (ODE) problem (Eq. (11)),

$$
\frac{d Q}{d t}=-\frac{1}{V_{i}} C\left(Q_{i}\right) \text {, }
$$

discretized using a fully explicit second-order accurate, five-stage Runge-Kutta time stepping scheme to advance the solution of the governing equations in time. The scheme can be written as

$$
\begin{aligned}
& Q_{i}^{(0)}=Q_{i}^{n}, \\
& Q_{i}^{(1)}=Q_{i}^{(0)}-\alpha_{1} \frac{\Delta t}{V_{i}} C\left(Q_{i}^{(0)}\right), \\
& \vdots \quad \vdots \\
& Q_{i}^{(5)}=Q_{i}^{(0)}-\alpha_{5} \frac{\Delta t}{V_{i}} C\left(Q_{i}^{(4)}\right), \\
& Q_{i}^{n+1}=Q_{i}^{(5)},
\end{aligned}
$$

where the superscripts $n$ and $n+1$ denote respectively the values of the properties at the beginning and at the end of the $n-t h$ time step. As suggested by Mavriplis, (1988), the $\alpha$ coefficients are $\alpha_{1}=1 / 4, \alpha_{2}=1 / 6, \alpha_{3}=3 / 8, \alpha_{4}=1 / 2$, and, $\alpha_{5}=1$.

\section{ENO AND WENO RECONSTRUCTION}

The reconstruction procedure of the ENO schemes is based on the approximation of mean values of the primitive variables for each cell in the mesh by polynomials of one order less than the spatial order of accuracy expected. For the construction of polynomials of $\eta-t h$ order, one must use $N(\eta)=(\eta+1)(\eta+2) / 2$ cells. The first step in obtaining the polynomial reconstruction for each cell is to define the possible set of cells, called stencil, that will be used. In the finite volume cell centered scheme the stencils can be selected in a Von Neumann neighborhood for a linear polynomial reconstruction, as shown in Fig. 1.

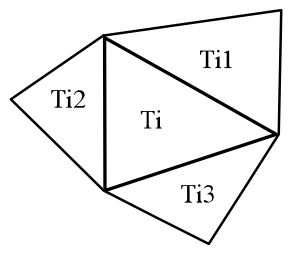

Figure 1. Von Neumann neighborhood of the Ti triangle.

As only a maximum of three polynomials can be reconstructed by the use of three neighbors, there may be problems due to overshoots and undershoots with such a reconstruction. The option adopted in this work follows the ideas of Sonar, (1997), which are to amplify the number of possible reconstructed polynomials using the neighbor cells, in the sense of cells that share a common edge, of the Von Neumann neighborhood as indicated in Fig. 2. Therefore, the number of calculated polynomials is six for the cells in regions far from the boundaries of the grid because the stencils for the cases where one has two neighbor cells of the $T_{i}$ triangle are not being considered in the calculations. In the cells that share a common edge with a ghost volume the stencils are constructed using a Von Neumann neighborhood as shown in Fig. 1. 


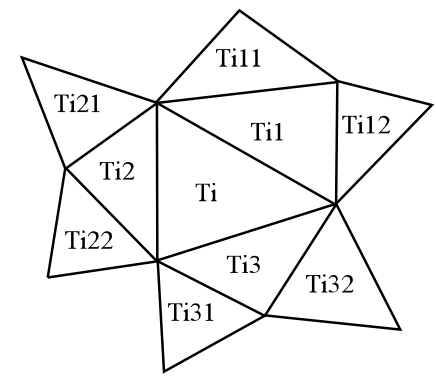

Figure 2. Neighborhood of Ti actually utilized in the computations.

In the present work, the cells are simply triangles and the $p(x, y)$ polynomials can, then, be calculated as the following formulation

$$
p(x, y)=\sum_{|\beta| \leq n} r_{\beta 1 \beta 2}\left(x-x_{c}\right)^{\beta 1}\left(y-y_{c}\right)^{\beta 2}
$$

where $|\beta|=\beta_{1}+\beta_{2}$, with $\beta_{i} \in\{1,2,3, \ldots\}, x_{c}$ and $y_{c}$ are the Cartesian coordinates of the barycenter of the triangle and $r_{\beta 1 \beta 2}$ are unknown coefficients that must be determined.

Once it is established that $p(x, y)$ is a good approximation to the mean values of primitive variables for each cell one can write a linear system, $[R]\{r\}=\{\bar{u}\}$, of $N(\eta)$ equations for the $N(\eta)$ unknowns $r_{\beta 1 \beta 2}$. Here, $[R]$ is the matrix of control volume moments as in Gooch, (1997), computed using a scaling technique proposed by Friedrich, (1998) to circumvent a poorly conditioned matrix. Moreover, $r$ is the vector of unknown $\beta$ 's and $\bar{u}$ is the vector with the mean values for each primitive variable. The stencil is considered admissible if $[R]$ matrix is invertible. There are other approaches for the calculation of the polynomials, as the one proposed by Abgrall, (1994), which uses interpolation through barycentric coordinates, or the one proposed by Hu and Shu, (1999), which is based in reconstructing high order polynomials using only linear polynomials. These alternate approaches, however, are beyond the scope of the present paper.

After the polynomial reconstruction is performed for each cell, the next step is to verify which polynomial is the less oscillatory to use in the ENO scheme. The oscillation is computed using some indicator that assesses the smoothness of $p(x, y)$. Abgrall, (1994) proposed the oscillation indicator defined as

$$
O I_{A b}=\sum_{|\beta|=\eta}\left|r_{\beta 1 \beta 2}\right|,
$$

which is the absolute value of the summation of the highest degree polynomial coefficients. Harten and Chakravarthy, (1991) proposed the indicator given by

$$
O I_{H C}=\sqrt{\sum_{n=1}^{\eta} \sum_{|\beta|=n} r_{\beta 1 \beta 2}^{2}} .
$$

Jiang and Shu, (1996) proposed an indicator, which was later modified by Friedrich, (1998), and that can be expressed as

$$
O I_{J S}=\sqrt{\left(\sum_{1 \leq|\beta| \leq n} \int_{V_{i}} h^{2|\beta|-4}\left(\frac{\partial^{|\beta|} p(x, y)}{\partial x^{\beta 1} \partial y^{\beta 2}}\right)^{2} d x d y\right)} .
$$

The first two oscillation indicators were tested and compared in the present work.

Differently from the ENO schemes, the WENO schemes use all the calculated polynomials. These polynomials are added together through the use of weights which are computed for each one of the polynomials as proportional to its respective oscillation indicator. The main idea in the WENO reconstruction is to attribute the computed weights for each polynomial with the aim of reconstructing a new polynomial as $p(x, y)=\sum_{k=1}^{m} \omega_{k} p_{k}(x, y)$. The weights are of order one in the smooth regions of the flow and are of order $h^{m}$ in the regions with discontinuities, where $h$ is the mesh width and $m$ is the number of admissible stencils for the reconstruction of $p(x, y)$. The weights can be computed as

$$
\omega_{k}=\frac{\left[\varepsilon+O I\left(p_{k}(x, y)\right)\right]^{-\theta}}{\sum_{k^{\prime}=1}^{m}\left[\varepsilon+O I\left(p_{k^{\prime}}(x, y)\right)\right]^{-\theta}},
$$

where $\varepsilon$ is a small real number used to avoid division by zero and $\theta$ is a positive integer.

\section{RESULTS AND DISCUSSION}

The present section discusses computational results which were obtained with the objective of verifying the new implemented capability and assessing its advantages/disadvantages with regard to the discretization methods previously available in the code. Hence, the test cases here analyzed were selected among those for which analytical solutions and/or well document, independent data are available in the literature.

The first case shown is a shock tube problem with lenght 10.0 and height 1.0 dimensionless units. The entire mesh, with 4697 nodes and 8928 volumes, and a detailed part of it are shown in Fig. $3 \mathrm{a}$ and $3 \mathrm{~b}$, respectively. The results presented here are for pressure ratios, $p 1 / p 4$, of 5.0 and 100.0. Here, $p 1$ denotes the 
initial static pressure in the driver section (high-pressure side) of the shock tube, whereas $p 4$ denotes the corresponding initial static pressure in the driven section (low-pressure side) of the shock tube. In both test cases, density was made dimensionless with respect to the initial density and pressure was made dimensionless with respect to the characteristic speed of sound, both with respect to the driver section of the shock tube. It was assumed that both sides of the shock tube were originally at the same temperature.

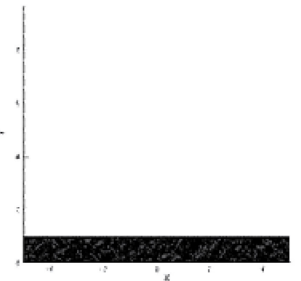

Figure 3a. Entire mesh for the shock tube problem.

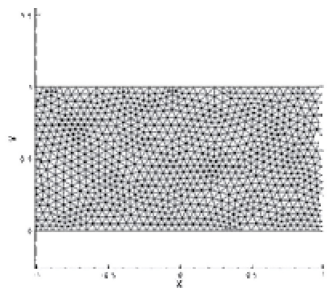

Figure 3b. Detail of the mesh for the shock tube problem.
Figures 4 and 5 present the results for the flow in the shock tube with initial pressure ratio of $p 1 / p 4=5.0$ for an instant of time equal to $1.0 \mathrm{sec}$. after diaphragm rupture. The results are presented in terms of density and Mach number distribution along the shock tube centerline. The results are ploted for the second-order ENO and WENO schemes implemented with different oscillation indicators and for the van Leer scheme with multi-dimensional minmod limiter (Azevedo, 1998). The analytical solution is also ploted with the purpose of comparing the accuracy of the numerical solutions.

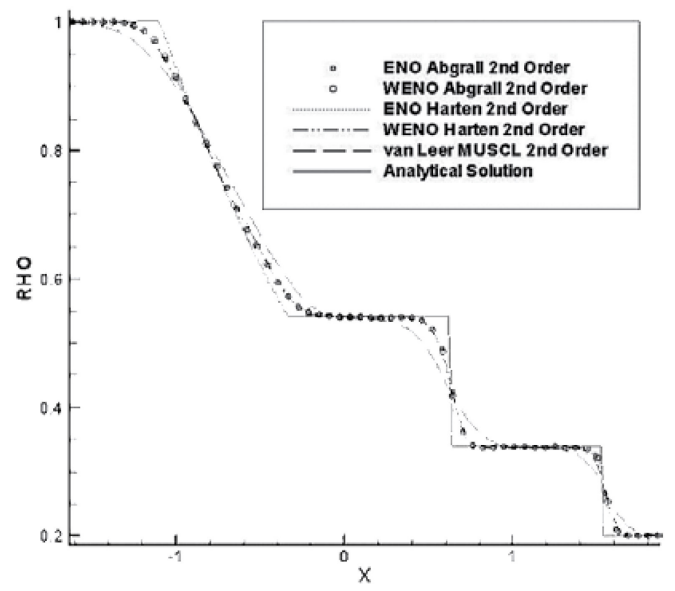

Figure 4. Comparison among ENO, WENO and van Leer MUSCL second-order methods for the shock tube problem with pressure ratio $\mathrm{p} 1 / \mathrm{p} 4=5.0$ at $\mathrm{t}=1.0 \mathrm{sec}$. after diaphragm rupture. Density along the centerline of the shock tube.

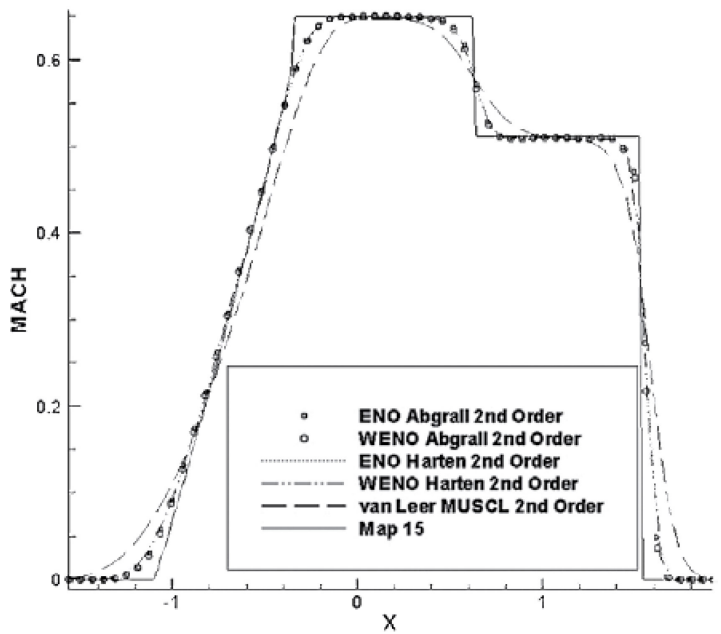

Figure 5. Comparison among ENO, WENO and van Leer MUSCL second-order methods for the shock tube problem with pressure ratio $\mathrm{p} 1 / \mathrm{p} 4=5.0$ at $\mathrm{t}=1.0 \mathrm{sec}$. after diaphragm rupture. Mach number along the centerline of the shock tube.

In this problem, a normal shock wave moves from the driver section of the shock tube to the driven section. As the normal shock wave propagates to the right with some velocity, it increases the pressure and induces a mass motion of the gas behind it. The interface between the driver and driven gases is represented by a contact discontinuity. An expansion wave propagates to the left, smoothly and continuously decreasing the pressure in driver section of the shock tube. All these physical phenomena are well captured by ENO and WENO schemes as can be viewed in Figs. 4 and 5.

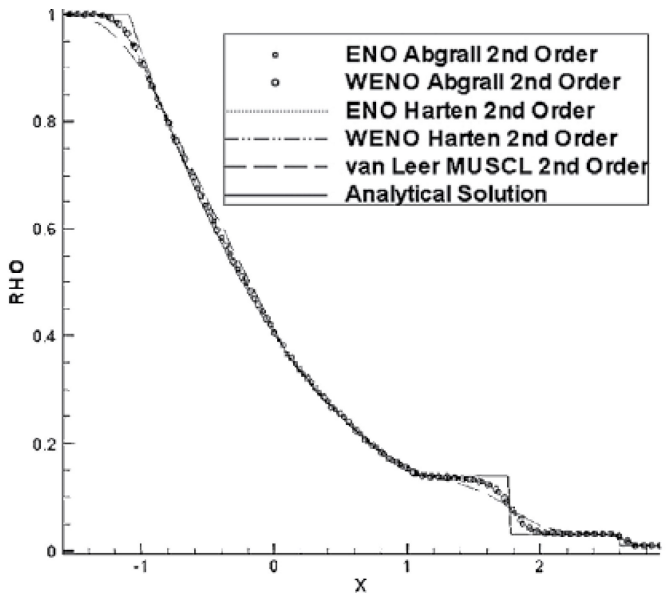

Figure 6. Comparison among ENO, WENO and van Leer MUSCL second-order methods for the shock tube problem with pressure ratio $\mathrm{pl} / \mathrm{p} 4=100.0$ at $\mathrm{t}=1.0 \mathrm{sec}$. after diaphragm rupture. Density along the centerline of the shock tube. 


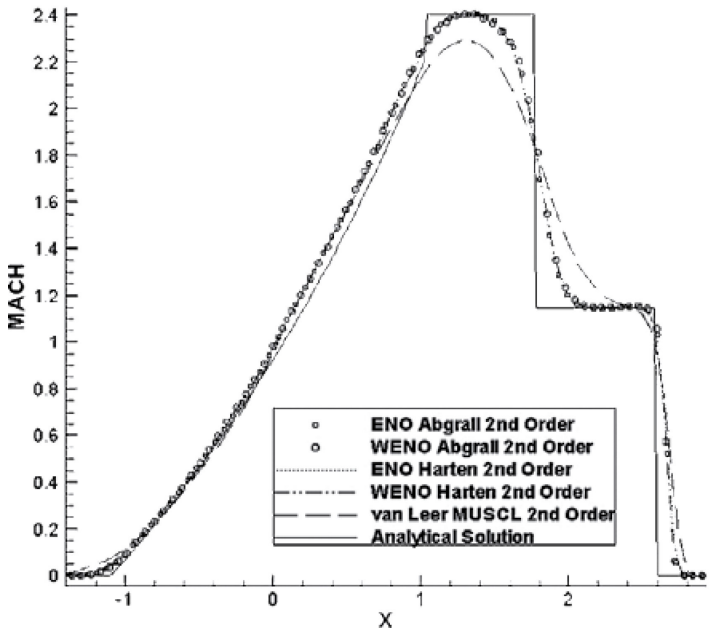

Figure 7. Comparison among ENO, WENO and van Leer MUSCL second-order methods for the shock tube problem with pressure ratio $\mathrm{p} 1 / \mathrm{p} 4=100.0$ at $\mathrm{t}=1.0 \mathrm{sec}$. after diaphragm rupture. Mach number along the centerline of the shock tube.

The ENO and WENO schemes are less diffusive than the van Leer scheme. Such a conclusion can be readily reached by observing the sharper representation of the shock wave and of the contact discontinuity. Figures 6 and 7 present the results for a pressure ratio of $p 1 / p 4=100.0$ for an instant of time equal to $1.0 \mathrm{sec}$. after diaphragm rupture. The results are presented in terms of density and Mach number distribution along the shock tube centerline.

Due to the very high pressure ratio in this case, one can observe that capturing of the phenomena by ENO and WENO schemes is less accurate than in the later case. However, both schemes are more accurate than the van Leer scheme. For the density plot, one can see that the van Leer scheme could not capture either the contact discontinuity nor the shock wave. The ENO and WENO schemes have, at least, captured the correct qualitative behavior, despite the rather diffusive appearance of both discontinuities. For the Mach number plot, both ENO and WENO schemes have captured the correct intensity of the Mach number in the contact discontinuity region and the van Leer scheme was not able to do it. Furthermore, one can also observe in Figs. 4 to 7 that the results with either the ENO or WENO schemes have shown no effects of the form of the oscillation indicator used. As previously discussed, the work here reported has implemented both Abgrall, (1994) and Harten and Chakravarthy, (1991) oscillation indicators. Hence, in view of these results, the forthcoming calculations will consider only the oscillation indicator proposed by Abgrall.
The second test case is a compressible flow in a channel with a forward-facing step that is well documented in Woodward and Colella, (1984). The channel lenght is 3 with height 1 dimensionless units. The step is located in a position 0.6 dimensionless units from the channel entrance. It has a height of 0.2 dimensionless units. The entire mesh has 7022 nodes and 13608 volumes. A complete view of this mesh, together with a blow-up view of the step region, can be seen in Figs. $8 \mathrm{a}$ and $8 \mathrm{~b}$.

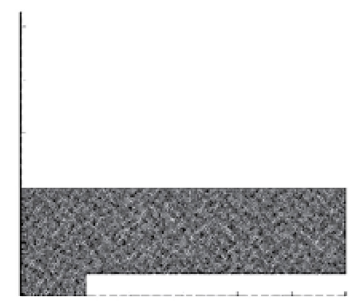

Figure 8a. Entire mesh for the channel.

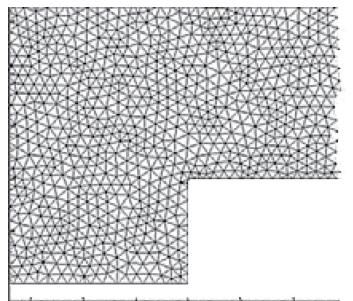

Figure $8 b$. Detail of the mesh in the step corner region.
An uniform Mach number of 3.0 inflow is set at $\mathrm{t}=0 \mathrm{sec}$. for a gas with density made dimensionless with respect to the entrance conditions and pressure made dimensionless with respect to density times speed of sound squared. These properties were made dimensionless as in Abgrall, (1994). One should observe that this test case is unsteady. Results at $\mathrm{t}=1.5 \mathrm{sec}$. are plotted in terms of density contour lines in Fig. 9 for the ENO scheme and in Fig. 10 for the WENO scheme. At this instant of time, a detached shock is formed in front of the step and it reflects first in the upper surface of the channel and, second, in the upper surface of the step. As can be observed in Woodward and Colella, (1984), the positions of the shock and shock reflections are the same. However, their capture is sharper in the cited reference due to a more refined mesh. A slip line that appears due to the interaction between the shock created by the expansion wave at the corner and the reflected shock is captured in the present computations with a bit more smearing than in the cited reference.

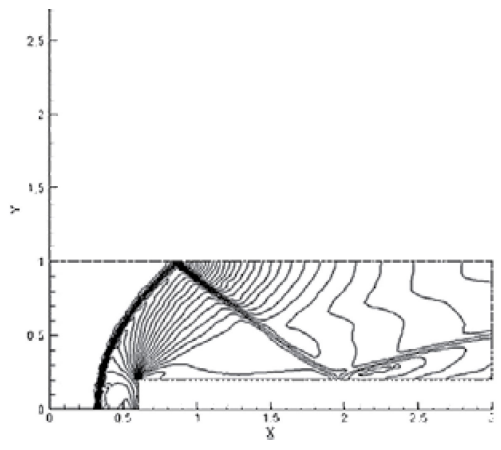

Figure 9. Density contours for the flow in the channel for the ENO method at $\mathrm{t}=1.5 \mathrm{sec}$. 


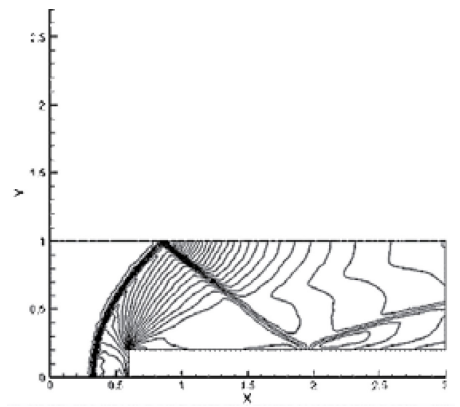

Figure 10. Density contours for the flow in the channel for the WENO method at $\mathrm{t}=1.5 \mathrm{sec}$.

The more interesting structure of the flow develops at time equal to $t=4.0 \mathrm{sec}$. Results at this instant of time appear in Fig. 11 for the computation with the ENO scheme and in Fig. 12 for the computation with the WENO scheme. In both figures, the density contour lines are presented. At this instant of time, the detached shock evolves to a lambda shock that reflects in the upper surface of the step, and this shock moves upstream with respect to the shock reflection position at $\mathrm{t}=1.5 \mathrm{sec}$. Since the first reflection, in these cases, occurs further upstream, there is still another shock reflection on the upper wall of the channel within the present computational domain.

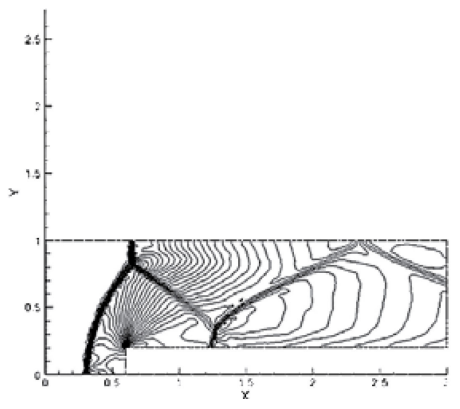

Figure 11. Density contours for the flow in the channel for the ENO method at $\mathrm{t}=4.0 \mathrm{sec}$.

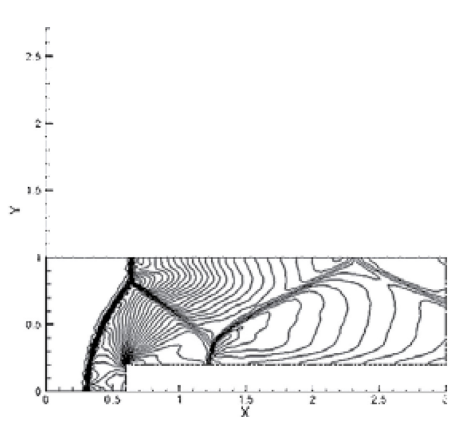

Figure 12. Density contours for the flow in the channel for the WENO method at $\mathrm{t}=4.0 \mathrm{sec}$.
This behavior is different from what can be seen in Figs. 9 and 10 for $t=1.5 \mathrm{sec}$., and it is in agreement with the results in Abgrall, (1994) and Sonar, (1997). The slip line that appears coming from the lambda shock can be seen in the density contour lines. The positions of the shocks are in good agreement with the data available in Woodward and Colella, (1984), despite the fact that they are better captured in the cited reference and that in the present work no special treatment was applied to the corner step region. In general the results are very similar to those presented in Abgrall, (1994) for second and third order ENO schemes and Sonar, (1997) for second order ENO schemes.

The last test case considered here is the transonic flow over a NACA0012 airfoil with zero degree angle-of-attack. The freestream has a Mach number value of $M_{\infty}=0.8$. The pressure and density were made dimensionless with respect to the freestream conditions. This case was computed using an unstructured mesh with 4369 nodes and 8414 volumes and one can see a detail of it in Fig. 13.

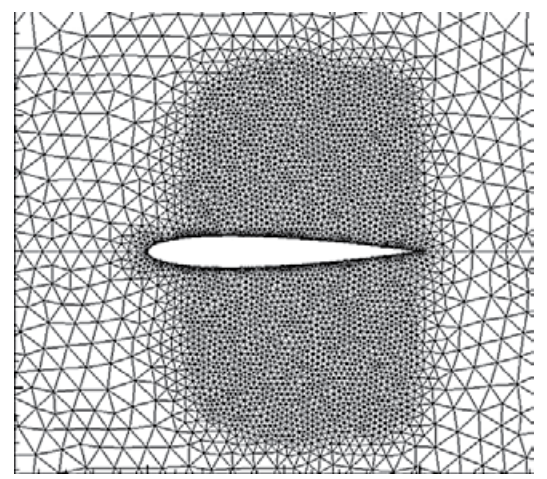

Figure 13. Detail of the mesh for the NACA0012 airfoil.

This is a steady case and, here, the CFL number was set as a constant value. Therefore, differently from the previous unsteady computations in which the time step was set constant throughout the flowfield, the CFL number is kept constant through the flow in the present steady computation. Hence, one sets the CFL number and the local time step is computed using the local grid spacing and characteristic speeds. The numerical results are ploted in Fig. 14 for pressure contours and in Fig. 15 for the $\mathrm{Cp}$ distribution along the airfoil chord using the WENO scheme and for an experimental test obtained from Scalabrin, (2002).

One can observe the accuracy of the method in capturing the shock over the airfoil. The circles represent the experimental data and the triangles represent the results obtained with the WENO scheme. 


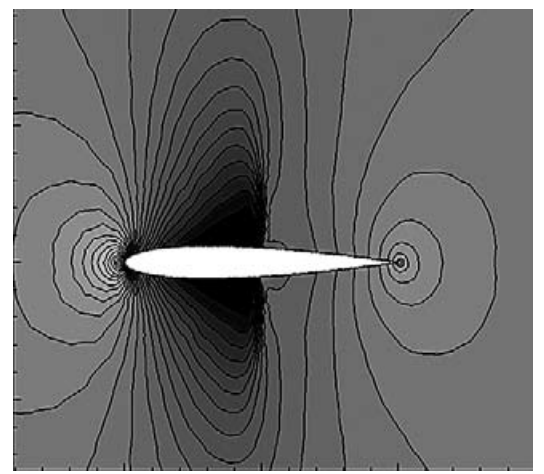

Figure 12. Pressure contour over the NACA0012 airfoil NACA0012 airfoil for $\mathrm{M}_{\infty}=0.8$ and $\alpha=0$.

\section{CONCLUSIONS}

The reconstruction of essentially non-oscillatoty (ENO) schemes and weighted essentially non- oscillatoty (WENO) schemes is presented in this work with some results that have shown the accuracy of the methods. The theoretical formulation included the spatial discretization and the temporal discretization, with details of the ENO and WENO

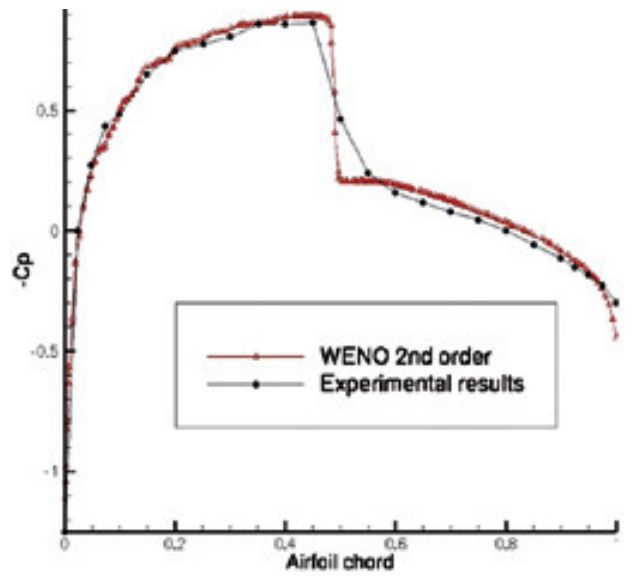

Figure 13. Cp distribution along the chord of a NACA0012 airfoil for $\mathrm{M}_{\infty}=0.8$ and $\alpha=0$.

reconstruction in a generic framework to facilitate the use of polynomials of any order of accuracy. Nevertheless, in this paper, only second-order accurate schemes are shown. The cost of the ENO and WENO schemes is almost the same, because the additional work required for a WENO implementation is the computation of the weights and of the new polynomial, which is negligible compared to the overall cost of a time step. The cost of the van Leer method is about three times smaller than the cost of the ENO or WENO methods.
Three test cases are studied in this work with the purpose of comparing the results for ENO and WENO schemes with analytical data, experimental data or other second-order methods already tested and validated by the group. The first test case studied is the classical shock tube problem for two different pressure ratios. The unsteady flow in a channel with a forwardfacing step, well documented in Woodward and Colella, (1984), and the transonic steady flow over a NACA0012 airfoil are also studied. The ENO and WENO schemes have shown to be more accurate capturing the discontinuities appearing in the test cases analyzed. Future work will consider higher order reconstruction through the implementation of third-order and fourth-order ENO and WENO schemes. As discussed, however, the framework for such implementation is already available with the work presented in this paper.

\section{ACKNOWLEDGMENTS}

The authors gratefully acknowledge the support of Fundação de Amparo à Pesquisa do Estado de São Paulo FAPESP through a Masters Scholarship for the first author under the grant 03/10047-2.

\section{REFERENCES}

Abgrall, R, 1994, On Essentially Non-Oscillatory Schemes on Unstructured Meshes: Analysis and Implementation, Journal of Computational Physics, Vol. 114, pp. 45-58.

Azevedo, J., L. F. and Korzenowski, H., 1998, Comparison of Unstructured Grid Finite Volume Methods for Cold Gas Hypersonic Flow Simulations. In: Proceedings of the 16th AIAA Applied Aerodynamics Conference, AIAA Paper No. 98-2629, pp. 447-463.

Friedrich, O., 1998, Weighted Essentially Non-Oscillatory Schemes for the Interpolation of Mean Values on Unstructured Grids, Journal of Computational Physics, Vol. 144, pp. 194-212.

Gooch, C. F. O., 1997, High-Order ENO Schemes for Unstructured Meshes Based on Least-Squares Reconstruction, Argonne National Laboratory, Report No. P631-1296.

Harten, A., Chakravarthy, S. R., 1991, Multi-Dimensional ENO Schemes for General Geometries, ICASE Report No. 91-76.

Harten, A., Osher, S., Engquist, B., and Chakravarthy, S. R., 1987, Uniformly High Order Accurate Essentially NonOscillatory Schemes III, Journal of Computational Physics, Vol. 71, pp. 231-303.

Hu, C., and Shu, C. W., 1999, Weighted Essentially Non-Oscillatory Schemes on Triangular Meshes, Journal of Computational Physics, Vol. 150, pp. 97-127.

Jiang, S., and Shu, C. W., 1996, Efficient Implementation of Weighted ENO Schemes, Journal of Computational Physics, Vol. 126, pp. 77-99. 
Liu, X. D., Osher, S, Chan, T., 1994, Weighted Essentially Non-Oscillatory Schemes, Journal of Computational Physics, Vol. 115, pp. 200-212.

Mavriplis, D. J., 1988, Multigrid Solution of the Two Dimensional Euler Equations on Unstructured Triangular Meshes, AIAA Journal, Vol. 26, pp. 824-831.

Roe, P. L., 1981, Approximatte Riemann Solvers, Parameter Vectors, and Difference Schemes, Journal of Computational Physics, Vol. 43, pp. 200-212.

Scalabrin, L. C., 2002, Numerical Simulation of ThreeDimensional Flows over Aerospace Configurations, Master Thesis, Instituto Tecnológico de Aeronáutica.

Sonar, T., 1997, On the Construction of Essentially NonOscillatory Finite Volume Approximations to Hyperbolic Conservation Laws on General Triangulations: Polynomial Recovery, Accuracy and Stencil Selection, Comput. Methods Appl. Mech. Engr., Vol. 140, pp. 157-181.

Woodward, P., and Colella, P., 1984, The Numerical Simulation of Two Dimensional Fluid Flow with Strong Shocks, Journal of Computational Physics, Vol. 54, pp. 115-173.

Received: September 06, 2006 Revised: October 06, 2006

Accepted: November 06, 2006 